\title{
Hot electron science in plasmonics and catalysis: what we argue about
}

\author{
Jeremy J. Baumberg*1 \\ ${ }^{1}$ NanoPhotonics Centre, Cavendish Laboratory, Department of Physics, JJ Thompson Avenue, University of \\ Cambridge, Cambridge, CB3 OHE, UK
}

\begin{abstract}
Hot electron photochemistry has made strong claims for improved control or chemical reactions. Here we discuss these claims in the light of a plethora of model experiments and theories, asking what are the key issues to solve. I particularly highlight the need to understand nanoscale thermal hot-spots, thermal gradients, and thermal transport, as well as the conventional optical confinement in plasmonics. I note how the 'direct electron transfer' process seems to dominate, and resembles well known 'indirect excitons' in semiconductor quantum wells. I believe a crucial advance still required is a prototype nano-confined geometry which allows reactants and products to access a well-controlled metallic atomic surface.
\end{abstract}

KEYWORDS: Plasmonics, Catalysis, Nanocavities, Hot electrons,

\section{Introduction}

I started this meeting with optimistic views about the possibility of tackling several puzzles, paradoxes, and propaganda that have accreted around the field of hot electrons in plasmonics. While this meeting has approached some of these, and cleared away some of the vegetation to allow a better view, many of the issues remain implacably present, and the aim here is to present these in some context.

This has been a fascinating meeting, with perspectives on the concepts and utilisation of hot electrons for harnessing from a variety of directions. In this summary, I will discuss these in terms of models, of experiments, and of the probes used. I then aim to point to some areas of terminology that the field should harmonise, before discussing the challenges and some possible approaches to tackle them.

\section{Prior experience}

I would start by outlining a few recent experiments from my own research group that have suggested the influence of hot electrons, before critiquing them. One approach that we have used is to explore polymerisation reactions that can be initiated by the hot electrons from plasmonic nanoparticles (NPs). This has utilised the Nanoparticle-on-Mirror or NPoM (also sometimes called particle-over-film or other descriptions, see ${ }^{1}$ and below) immersed in pure monomers of radical polymerisation such as divinylbenzene (DVB), styrene (St), or methyl methacrylate (MMA). Our aim in this work is to avoid complications from oxides by using only $\mathrm{Au}$, and preventing Schottky barrier effects by eliminating semiconductor layers such as $\mathrm{ZnO}$ or $\mathrm{TiO}_{2}$, to avoid any competing photochemistries. We mix quenchers into the monomer, and omit the radical initiators normally used to start the reaction. When illuminated (which is most efficient for wavelengths tuned to the trapped plasmon in the nanometre gap between the spherical nanoparticle and the planar mirror), hot electrons create radicals only at the metal surface, and these initiate the polymerisation which terminates within $20-50 \mathrm{~nm}$ of the 
surface ${ }^{2,3}$. Upon washing away unreacted monomer, this leaves polymer surface-coating the NP around the gap (Fig.1). The growing polymer forces the NP above the mirror surface, thus tuning the gap plasmon, and allowing the process to be controlled in real time by monitoring the dark-field scattering of the plasmonic construct. The hot electrons thus allow control of where monomers crosslink, in a similar way to conventional UV photoresists.
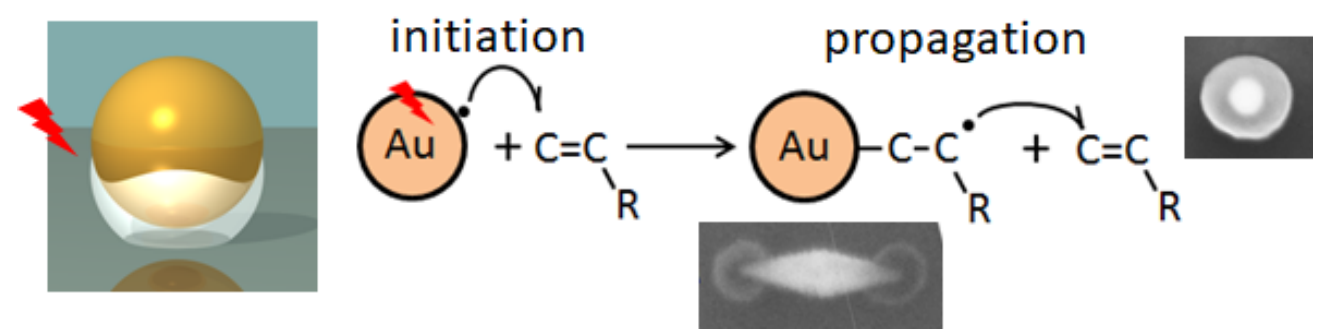

Fig.1: Plasmon-induced radical polymerisation, illuminating a single NPoM with $\mu \mathrm{W} C W$ light immersed in monomers, from $[2,3]$.

A second series of experiments (Fig.2) uses the same NPoM geometry but inserts a few redoxactive molecules into the gap whose oxidation state can be measured in real-time by the surfaceenhanced Raman scattering (SERS) of their vibrations. When the electronic state changes, the modified electron density in the bonds changes their vibrational frequencies allowing remote tracking of their charge state, even at the single molecule level ${ }^{4}$. Essentially this allow us to watch chemistry in real time, with hot electrons jumping from the Au driving the process stochastically, depending on the conductivity of the molecule-metal interfaces. More recent experiments use bis-phthalocyanine nanogap spacers which are only $0.4 \mathrm{~nm}$ wide but contain single lanthanide ion cores that can tune the electronic and magnetic properties of the nanogap, thus changing the plasmonic mode ${ }^{5}$. The difficulty of full theoretical simulations that combine both the quantum mechanics of the molecular electronic states with the classical electromagnetism of the plasmonic confinement, means that such experiments cannot yet be accounted for by theory.

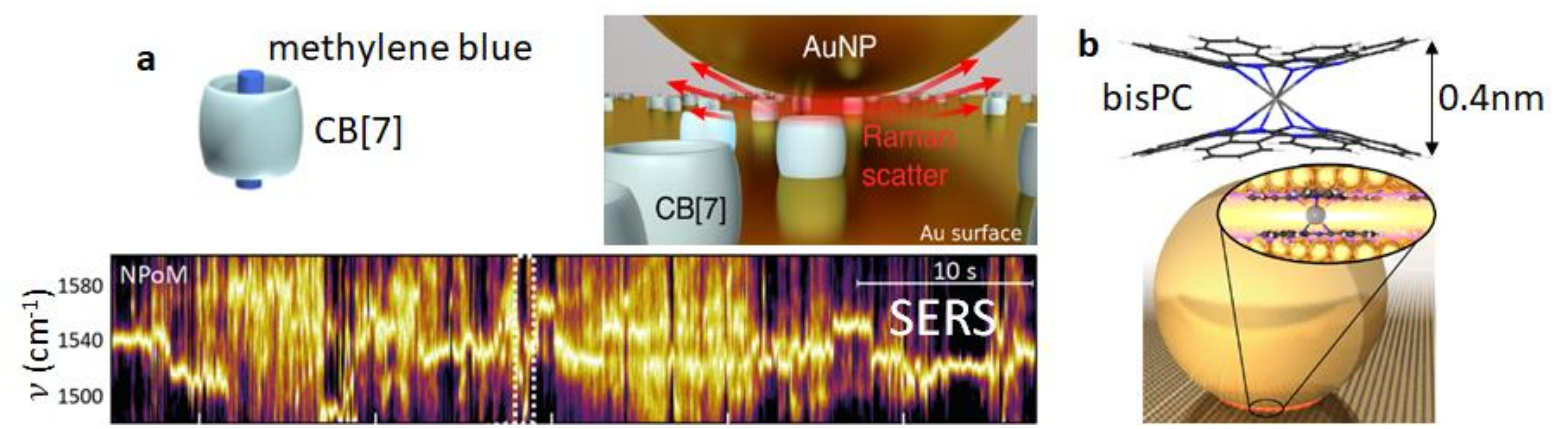

Fig.2(a): Single molecule redox, using methylene blue in CB[7] cage spacers within the NPoM nanogap, whose Raman emission (below) shows discrete jumps on single electron changes (from ${ }^{4}$ ). (b) Bisphthalocyanine spacer with lanthanide ion in centre $\left(\right.$ from $\left.{ }^{5}\right)$.

A final class of experiments uses light or voltage to change the redox state around such nanoconstructs, for instance reducing copper oxide nanoparticles back to plasmonic copper ${ }^{6}$ or creating tuneable wallpapers for building-scale display applications ${ }^{7}$. In this case electrons are involved in the redox processes but it is not clear if they can be termed hot or not.

Such experiments are perhaps typical examples of the enthusiasm for exploring hot electron effects within plasmonics. They can be critiqued more generally in that all hot electron effects can be 
inferred, it is extremely hard to understand the local phonon temperatures in the metals and molecules at the same time, even though they are well defined precise constructs. Most work in the field as we see at this meeting involves much less well defined geometries making the task of separating the causes of the observations much harder. A theme of this summary will be that we need to find new constructs that allow full measurements and control of hot electrons in precise nanogeometries.
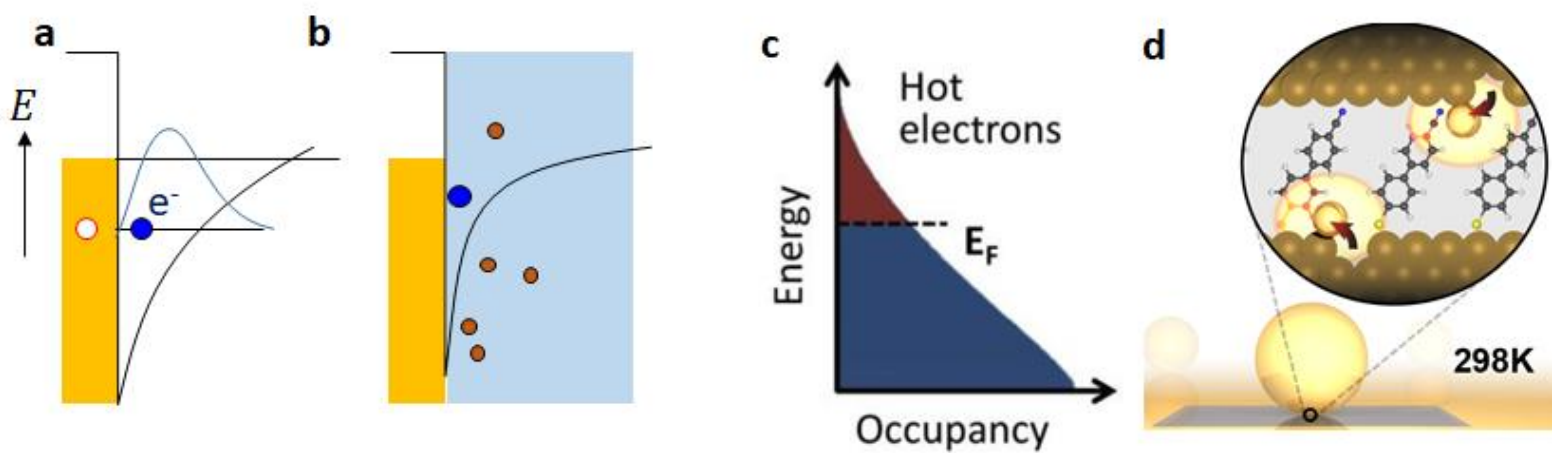

Fig.3. (a) Electron surface states outside metal, which (b) are screened with aqueous ions. (c) Hot Boltzmann distributions allow hot electrons to interact with surface states. (d) Gold atom movement tracked in real time in NPoMs ( from $^{8}$ ).

One particular issue is understanding the surface electronic states. Electrons at the surface of a metal such as gold experience their own image charges, thus forming an attractive potential above the surface which contains quantised states (Fig.3a). Outside a vacuum environment, these states are screened. Even in air, nanoscale morphologies instantly collect monolayer-thick water films with ions which heavily screen away such confinement (Fig.3b), however this is considerably altered by physisorbed and chemisorbed molecules. These combined surface electronic states are rarely characterised. A second discussion is what is meant by 'hot electrons': this can mean (a) non-thermal electrons, (b) thermalized electron temperatures greater than the lattice temperature, or (c) locally hot but still lattice-thermalized regions of electrons above the global temperature (Fig.3c). We will return to this discussion later. Thirdly, it is important to realise that metal nanoparticles below their volume melting temperature (which is already suppressed below the bulk metal melting point) all possess surface atomic layers with much higher mobilities, so their liquid-like surface atoms scurry around at room temperature. We have recently demonstrated that both at low temperature ${ }^{9}$ and room temperature ${ }^{8}$, plasmonic nanoparticle structures even at $\mu \mathrm{W}$ illumination powers exhibit dynamic surface reconstruction. The extremely tight plasmonic volumes allow the movement of even single Au or Ag atoms to be tracked in real time, reinforcing a picture in which binding of molecules to the surface is mostly transient and constantly reforming (Fig.3d).

\section{Models}

At this meeting we have seen a number of models for hot electron production at plasmonic surfaces (Fig.4), including a beautiful review of the field from Naomi Halas ${ }^{10}$. Basic analytic understanding ${ }^{11}$ allows us to see that the 'surface damping' (Fig.4a) dominates hot electron production. This is despite much calculation in the literature picking out the quantum absorption of plasmons to estimate hot electron production, and which generally has not been tested quantitatively against experiments. This process is similar to 'plasmon Bremstrahlung' (Fig.4b) which gives plasmon-enhanced light emission 
from the electronic-Raman scattering (ERS) process that depends on the plasmon field gradient at the surface of any nanostructure (see also ${ }^{12}$ ).

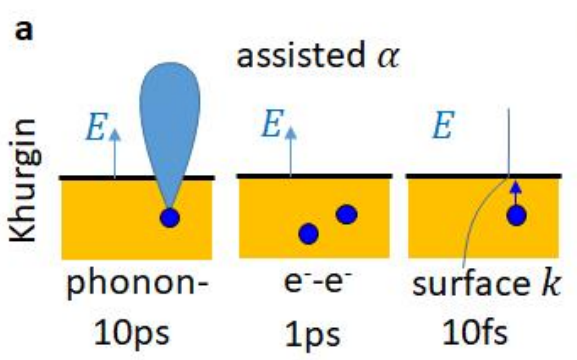

f

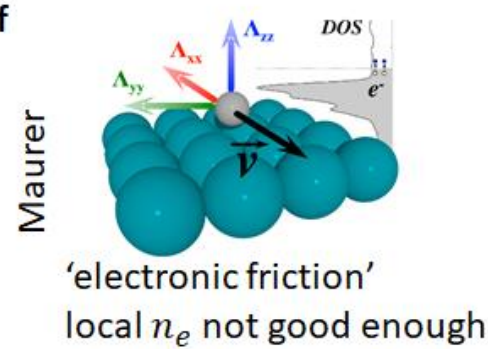

b

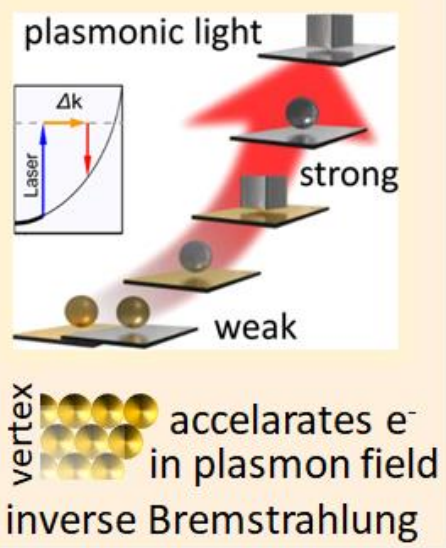

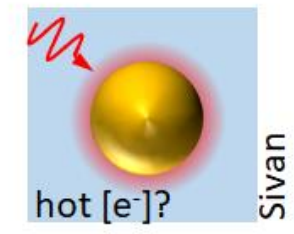

e
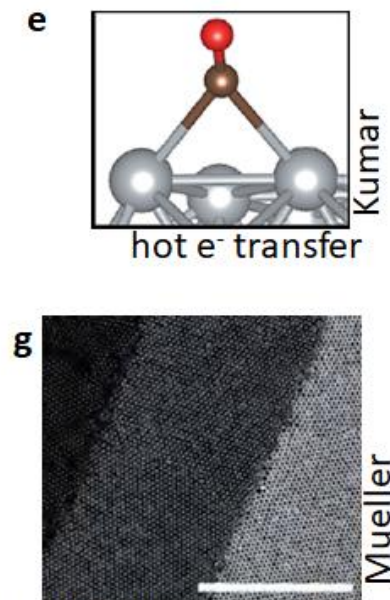

AuNP bilayers: higher $\alpha$

Fig.4. (a) Hot-e- timescales, needing different scattering processes. Only surface generated $\mathrm{e}^{-}$are significant ${ }^{11}$. (b) Model of electronic-Raman scattering (ERS) giving light emission at surfaces from the rapid spatial decay of plasmons, in a type of inverse Bremstahlung ${ }^{13}$, which will be enhanced at tip vertices around nanostructures. (c) Model of thermal heating on nanostructures in embedded thermal environments ${ }^{14}$. (d) Comparison of transfer of electrons across surface, and the indirect excitation of carriers in the near-field ${ }^{15}$. (e) Full calculation of indirect excitation of $\mathrm{e}^{-}$in $\mathrm{CO}$ on $\mathrm{Ag}$ nanocluster ${ }^{16}$. ( $\mathrm{f}$ ) Beyond using local surface electron density for molecular damping ${ }^{17}$. (g) Model system of bilayer of Au nanoparticles ${ }^{18}$.

A crucial discussion opened by Yonatan Sivan et al. $^{14}$ is the need for a proper analysis of the local temperature at the nanostructure surface, when illuminated (Fig.4c). We will return to this below, but it generated heated and interesting dialogues at the meeting. The proposal is that this analysis explains all purported plasmonic hot electron catalysis through highly localised thermal heating at hot spots. Since catalysis has energy barriers, reaction rates are exponential sensitive on this localised heating. One crucial point is that comparing the temperature measured by a sample thermometer when heating the entire sample thermally vs heating optically, is not enough. This is because complicated plasmonic nanostructures will absorb light in different locations and the temperature rise will depend on thermal Kapitza resistances at all interfaces that are not well quantified.

Besides the surface hot electrons (the weak ballistic electrons we now ignore as noted above), we should also consider enhanced near-field absorption in the surface-bound molecules (Fig.4d), as well as 'direct transfer' where an electron from the metal is excited into the LUMO level of a surface molecule, or vice versa for holes. Full DFT can be used to calculate electron yields in the molecule (Fig.4e), though these are not 'hot' since they are directly injected into the molecule. We will discuss later the (confusing) different terminologies for this type of process in different research fields. A 
further difficulty was highlighted of calculating damping effects on surface-bound molecules from metal underlayers (Fig.4f and ${ }^{17}$ ), while finally a nice model system was shown to form from bilayers of Au nanoparticles (Fig.4g).

\section{Experimental progress}

Many presentations at this meeting show intriguing materials nanostructures for enhancing catalytic interaction with plasmonic components, as well as different ways to characterise the hot electrons involved (Fig.5).

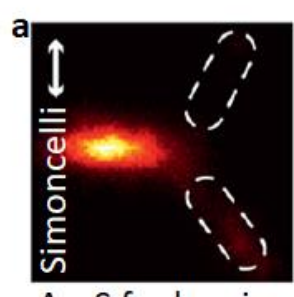

Au-S fs cleaving

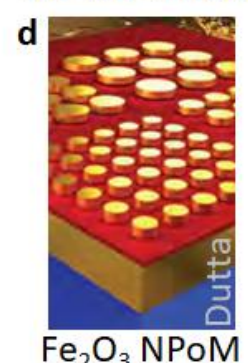

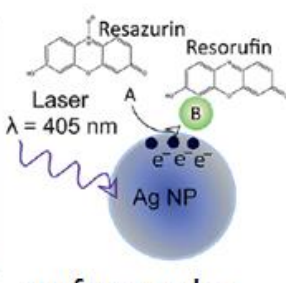

surface redox

e

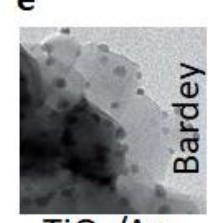

$\mathrm{TiO}_{2} / \mathrm{Au}$ b

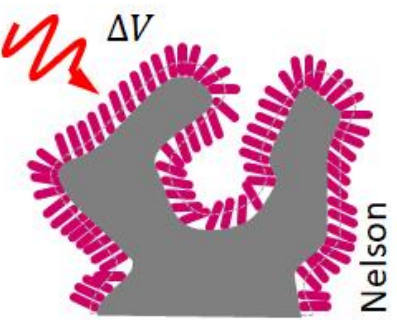

light-induced Stark shifts

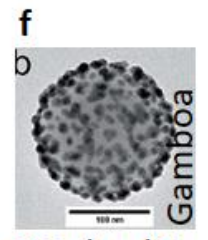

$\mathrm{SiO}_{2} / \mathrm{Au} / \mathrm{Pt}$ g

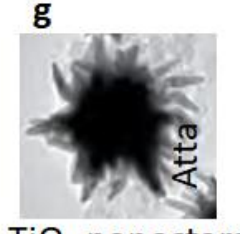

$\mathrm{TiO}_{2}$ nanostars

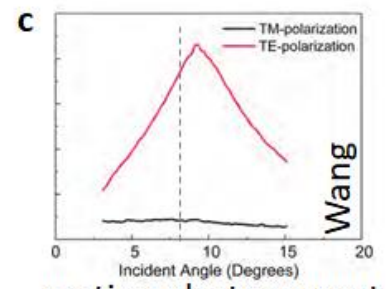

grating photocurrent
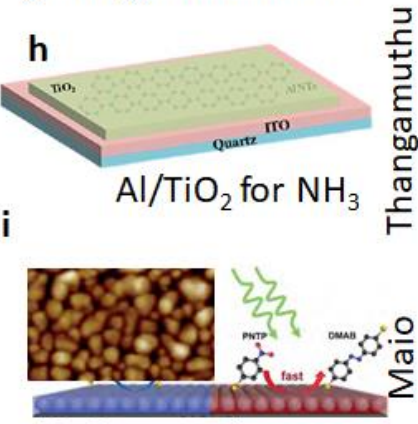

$\mathrm{MoS}_{2}$ coated

Fig.5. (a) Polarisation-dependent plasmons select Au-S bonds to cleave with fs pulses ${ }^{19}$, as well as studies of surface redox. (b) Measuring light-induced charging through Stark-shifts in SERS ${ }^{20}$. (c) Photocurrents produced at angled gratings ${ }^{21}$. (d) Enhancing photocatalytic efficiency in metalinsulator-metal geometries ${ }^{22}$. (e-i) Varieties of nanostructures for improving and enhancing photocatalysis ${ }^{23-27}$.

These involve different plasmonic metal/semiconductor interfaces in a variety of geometries, with predominantly Schottky barriers to harvest electrons from the mechanisms discussed above. In these cases the nanostructures are intricate but complex, and hence the precise quantitative understanding is rather difficult since the control is limited. Even for thin semiconductors inside arrays of metalinsulator-metal patches (Fig.5d) (or NPoM), it is hard for molecules to access the tightly confined gaps under the patches, so likely most photo-redox activity takes place at the edges of these patches. While optimisation for specific reactions is clearly of interest, without the understanding of electron yields at different facets and vertices, material development is not well focussed.

\section{Photocatalytic probes}

A third focus of this meeting has been the development of probes to be able to study the photocatalytic processes in more detail (Fig.6). One possibility is to use SERS gaps in the vicinity of the photocatalytic sites to track the reactants and products in real time (Fig.6a). A completely different approach is break bonds of an adsorbed molecule on a single-crystal surface watching where the 
reactants end up using STM (Fig.6b). Evaluating NPoM systems with different materials (Fig.6c), showed that absorption from lower quality plasmonic materials is not necessarily as much a problem in other plasmonic applications.

a

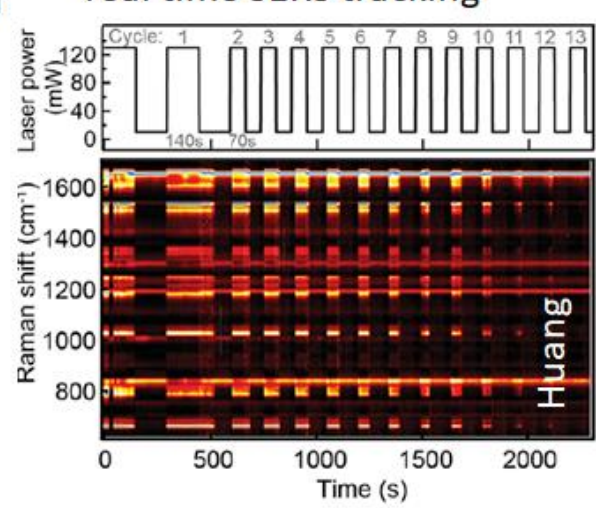

b

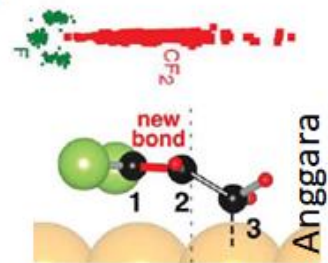

hot $\mathrm{e}^{-}$bond breaking molecule surface beam d

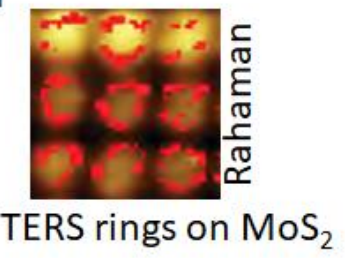

(d) $\mathrm{WSe}_{2}(\mathrm{AB})$

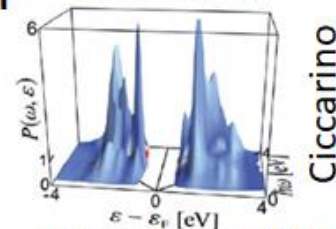

hot e-s from TMDs

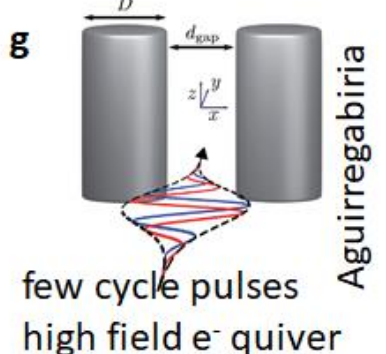

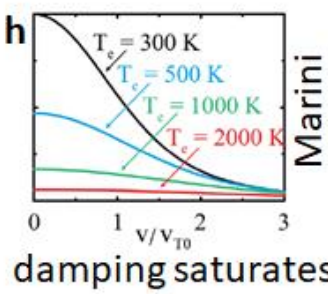

e $\mathrm{H}_{2}$ oxidation

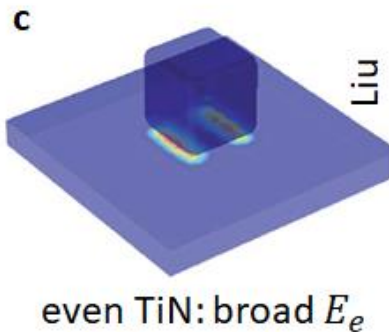

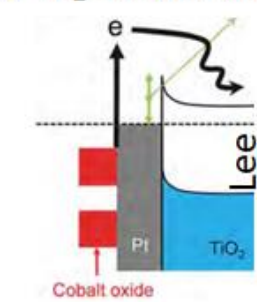

Fig.6. (a) SERS spectra as a function of time of reactants, intermediates, and products ${ }^{28}$. (b) STM tracking of molecular fragments ${ }^{29}$. (c) Cube on mirror (viz NPoM) geometries evaluated for photocatalytic activity with different material systems ${ }^{30}$. (d) $\mathrm{MoS}_{2}$ clad pillars show TERS enhanced in spatial halos ${ }^{31}$. (e) Cobalt oxide nanocubes on $\mathrm{Pt} / \mathrm{TiO}_{2}$ to quantify interface currents ${ }^{32}$. (f) Optimising ballistic hot electrons from TMDs ${ }^{33}$. (g) Quantifying nonlinear optical field driven electron currents ${ }^{34}$. (h) Nonlinear damping in metals ${ }^{35}$.

A further range of ideas were also presented at the meeting, using TERS (Fig.6d) and theory (Fig.6f) to consider TMD hot electrons, or controlling Schottky nanointerfaces (Fig.6e). Several theories also explored the nonlinear response of electrons, from the high intensity pulses in nanogaps (Fig.6g) and from fluid models of plasma transport (Fig.6h).

\section{Terminologies}

There are three among many overlapping terminological discussions that I will open up here. These arise because of the highly interdisciplinary nature of our community, and are a healthy sign of crossfertilisation of ideas, but also remain a confusing block to both younger and more experienced researchers as they are presented as separate or unknown processes, while in fact they can be slotted into much more established paradigms.

The first concerns the name of the process in which electrons from the metal are optically excited directly into the metal, so far referred to as 'direct transfer' (Fig.7a). This can also cover the excitation of an electron from the molecule being excited above the Fermi energy in the metal. This process has 
also been titled 'chemical-induced damping', presumably as near-surface electrons have a larger range of states to now be excited into, and may indeed by related to the surface damping concept added to the Drude model accounting for metal interfaces (but is not yet treated consistently within that framework). Another term is 'hybridisation' which describes how the spatial leakage of molecular wavefunctions into the metal, and spillout of electron wavefunctions from the metal allow the wavefunctions to mix together. This produces regions near the surface where both the leaking wavefunctions are co-located, thus allowing such optical transitions to occur at a single spatial site. Another term is 'plasmon resonant energy transfer' or PRET which describes dipole-dipole induced transfer of energy from donor to absorber mediated by (virtual) plasmons or metal-dressed photons, and I would argue that it is not clear how such a process where a plasmon is dipole-coupled to the molecular transition would be experimentally distinguished from the 'direct transfer' we have been discussing, apart from semantically. DFT calculations may useful in this respect, as shown in the meeting, but only if they can span systems large enough to hold the key physics. Finally, it is important to make a connection to a well-established (but apparently unknown to this community) similar scientific idea, which is unfortunately termed 'indirect absorption' known from semiconductor physics for $>30$ years. This forms exquisitely controllable potential landscapes for electrons using planar layers of different semiconductor materials typically grown by MBE and composed of III-V materials such as GaAs and AlGaAs alloys. Double quantum wells of width $5-20 \mathrm{~nm}$ can be grown with very thin barriers $(<5 \mathrm{~nm})$ in between which act as analogues for the metal and molecule systems here, since the wavefunctions leak through the barriers (Fig.7b). One optical transition is between a valance band to conduction band state in the same quantum well (direct exciton, or DX), and this is independent of a voltage applied between the quantum wells. A second transition is between the valence band in one quantum well and the conduction band in the neighbouring quantum well whose electronic wavefunction is exponentially leaking through the barrier, but provides some smaller oscillator strength. This is known in the field as a 'spatially-indirect exciton', but is identical to the 'direct electron transfer' here, and can be spectrally tuned by applying a voltage, as seen in many experiments ${ }^{36}$ (and references therein). Stark shifts are also sometimes seen for molecules on surfaces, but it is not easy to support a potential across this interface due to screening and conduction, but the spatially-indirect nature of the transition is well established and quantified exactly. I note that exactly the same spatially-indirect transition occurs for metal/semiconductor Schottky barriers which thus do not need to invoke ballistic transport.

a

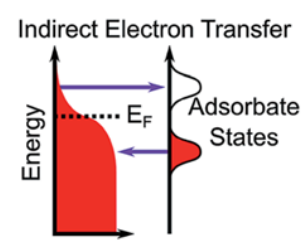

b

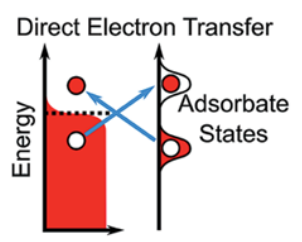

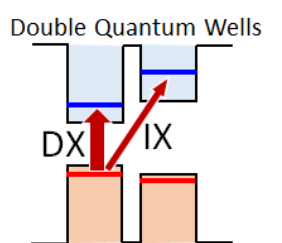

C free space cascaded coupling to localised plasmons

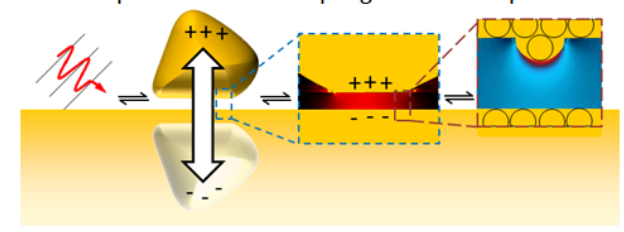

Fig.7: (a) Concepts of indirect (hot electrons in metal and ballistic transport) and direct electron transfer, from ${ }^{37}$. (b) Indirect exciton optical resonances in double quantum wells of semiconductor layers, from ${ }^{36}$. (c) Cascaded coupling of free space light into tightly localised plasmons, through plasmonic antennas, nanogaps, and even atomic protusions, from ${ }^{1}$. 
A second confusion is more trivial, and it is the profusion of names that have grown up around 'nanogap plasmons' which provide the most effective concentration of optical field. These range over the terms 'metal-insulator-metal' (MIM) waveguides, nanogap 'patch antennas', 'metastructures', and my own favoured 'nanoparticle-on-mirror' (NPoM). These all support similar plasmons, with a number of modes which can radiate more or less efficiently (see ${ }^{1}$ for a review and Fig.7c). The names reflect fabrication approaches from top-down to bottom-up, but remain somewhat confusing for those outside the field. The real trade-off here is between sharply spiked nanoparticles (from nanorods to nanostars) in which the asperities are hard to control and maintain in time but are highly accessible to reactants and allow products to escape, compared to nanogaps which have reliable and deep confinement of light but present steric barriers to reactants and products.

A third confusion in the meeting has been the discussion about what is a 'hot electron'. In the community, it is generally agreed that the vision of interest is to trigger photocatalytic reactions that are in some way different from those that are simply thermally driven. Just heating a catalytic nanoparticle sample creates a uniform temperature distribution, with minimal thermal gradients so that most surface reconstructions are static. Conversely irradiating with light typically causes highly localised heating enhanced by the plasmonic optical properties, and thus very inhomogeneous thermal distributions (literally 'thermal hot-spots') and high thermal gradients with significant dynamic surface atomic reconstruction. At the same time, it may also be possible for non-thermal electrons to be harvested by molecules adsorbed onto the plasmonic surfaces for chemical reactions that are otherwise not feasible, but these add onto the thermal hot-spot processes. Since catalysis is thermally activated, reaction rates are extremely sensitive to a small fraction of localised hot spots. I also emphasise that local lattice temperatures can dynamically exceed melting temperatures (one might term it 'superheating'), and certainly surface reconstruction takes place at much lower irradiations, and is optically catalysed ${ }^{9}$.

In this context, it is not clear that 'hot electrons' should only be those which are non-thermal (a very tiny fraction) or whose temperature exceeds the local lattice temperature. It is also not clear that we can call the direct electron transfer (which dominates over ballistic electron transport) 'hot' since it is a driven excitation of a two level system. However it is certainly plausible and observed that optical irradiation can enhance catalysis at a given substrate temperature, and change reaction pathways.

\section{Prospects}

In conclusion then, I would emphasise that while a huge amount of research has focussed on optical field enhancements in plasmonics, rather less effort has looked at the phonon transport in such nanostructures. The profusion of interfaces between dissimilar materials and hence the large Kapitza thermal resistances, the effects of morphology and nano-contacts on thermal transport, the effects of gas and solvent diffusion and convection in nanoscale pore geometries, and the effects of strong thermal gradients on atomic reconstructions that are crucial to provide step edges and facets that are active in catalysis, are all vital pieces of science to now study. The role of shape is subtle, since optical fields are localised around metal apexes, and penetration into the metal is enhanced at these points creating both thermal hot-spots and excited electrons in the metal and direct transfer to adsorbed molecules. Tight confinement at such points also gives large wavevector components that enhance absorption. It is thus likely that points and gaps are important in there photocatalytic effects, but they have not so far been well controlled. 
There is thus now a need for individual nanogeometries in which all these electromagnetic and thermal configurations are fully controlled. This would provide a testbed to investigate many of the issues raised here. These include influence of inhomogeneous temperatures, thermal transport, thermal gradient forces and potentials, direct and indirect electrons, shape, diffusion of species, reconstruction and degradation of activity, as well as their influence on a range of reactions. The latter is also a significant issue for the field, with a need to move beyond degradation reactions to bond formation. On the other hand, it is clear that surface reconstruction at the nanoscale depends on the molecular species being adsorbed, hence there is another subtle feedback where the hot electron/spot effects depend on the reaction being presented at the metal surface. The precise type of adsorption will also be important, with the orientation, proximity, atomic configuration of binding site, solvation, and screening which all affect hybridisation and wavefunction leakage that control electron/hole transfer to molecules.

Investigating such single nanostructures (or arrays of identical nanostructures) gives problems in detecting the small quantities of molecular product. While SERS techniques such as ${ }^{19,28}$ maybe help in some cases, more developments in this area are also needed, in solution phase as well as gas phase catalysis. The challenge of fabricating nanostructures remains significant, since both atomic scale and nanoscale to wavelength-scale features are all important. While I favour nanogap schemes such as the NPoM to provide some of this optical and structural control, the barrier remaining to solve is enabling molecular diffusion at this scale and control of facetting. Hence this research field has plenty of challenges for the next years that promise development of a real understanding of what is going on inside the photocatalytic (nano)box.

\section{AUTHOR INFORMATION}

\section{Corresponding Author}

*E-mail: (J.J.B.) jib12@cam.ac.uk

Notes

The authors declare no competing financial interest.

\section{ACKNOWLEDGMENT}

We acknowledge funding from the EPSRC Cambridge NanoDTC, EP/L015978/1 and EP/NO16920/1, and EP/L027151/1.

\section{References}

1. Baumberg, J. J., Aizpurua, J., Mikkelsen, M. H. \& Smith, D. R. Extreme nanophotonics from ultrathin metallic gaps. Nat. Mater. (2019). doi:10.1038/s41563-019-0290-y

2. Ding, T., Mertens, J., Lombardi, A., Scherman, O. A. \& Baumberg, J. J. Light-Directed Tuning of Plasmon Resonances via Plasmon-Induced Polymerization Using Hot Electrons. ACS Photonics 4, 1453-1458 (2017).

3. Wang, Y. et al. Plasmon-directed polymerization: Regulating polymer growth with light. Nano Res. 11, 6384-6390 (2018).

4. De Nijs, B. et al. Plasmonic tunnel junctions for single-molecule redox chemistry. Nat. Commun. 8, 1-7 (2017).

5. Readman, C. et al. Anomalously Large Spectral Shifts near the Quantum Tunnelling Limit in 
Plasmonic Rulers with Subatomic Resolution. Nano Lett. 19, 2051-2058 (2019).

6. Di Martino, G., Turek, V. A., Braeuninger-Weimer, P., Hofmann, S. \& Baumberg, J. J. Laserinduced reduction and in-situ optical spectroscopy of individual plasmonic copper nanoparticles for catalytic reactions. Appl. Phys. Lett. 110, 071111 (2017).

7. Peng, J. et al. Scalable electrochromic nano-pixels using plasmonics. Sci. Adv. (2019).

8. Carnegie, C. et al. Room-Temperature Optical Picocavities below $1 \mathrm{~nm} 3$ Accessing Single-Atom Geometries. J. Phys. Chem. Lett. 9, 7146-7151 (2018).

9. Benz, F. et al. Single-molecule optomechanics in "picocavities". Science. 354, 726-729 (2016).

10. Halas, N. J. Spiers Memorial Lecture: Introductory lecture: Hot-electron science and microscopic processes in plasmonics and catalysis. Faraday Discuss. (2019). doi:10.1039/C9FD00001A

11. Khurgin, J. B. Hot carriers generated by plasmons: where are they generated and where do they go from there? Faraday Discuss. (2019). doi:10.1039/C8FD00200B

12. García de Abajo, F. J. Optical excitations in electron microscopy. Rev. Mod. Phys. 82, 209-275 (2010).

13. Mertens, J., Kleemann, M.-E., Chikkaraddy, R., Narang, P. \& Baumberg, J. J. How Light Is Emitted by Plasmonic Metals. Nano Lett. 17, 2568-2574 (2017).

14. Sivan, Y., Un, I. W. \& Dubi, Y. Assistance of metal nanoparticles in photocatalysis - nothing more than a classical heat source. Faraday Discuss. (2019). doi:10.1039/C8FD00147B

15. Chavez, S., Rao, V. G. \& Linic, S. Unearthing the factors governing site specific rates of electronic excitations in multicomponent plasmonic systems and catalysts. Faraday Discuss. (2019). doi:10.1039/C8FD00143J

16. Kumar, P. V., Rossi, T. P., Kuisma, M., Erhart, P. \& Norris, D. J. Direct hot-carrier transfer in plasmonic catalysis. Faraday Discuss. (2019). doi:10.1039/C8FD00154E

17. Maurer, R. J., Zhang, Y., Guo, H. \& Jiang, B. Hot electron effects during reactive scattering of H 2 from $\mathrm{Ag}(111)$ : assessing the sensitivity to initial conditions, coupling magnitude, and electronic temperature. Faraday Discuss. (2019). doi:10.1039/C8FD00140E

18. Mueller, N. S. et al. Direct optical excitation of dark plasmons for hot electron generation. Faraday Discuss. (2018). doi:10.1039/C8FD00149A

19. Simoncelli, S. et al. Monitoring plasmonic hot-carrier chemical reactions at the single particle level. Faraday Discuss. (2019). doi:10.1039/C8FD00138C

20. Nelson, D. A. \& Schultz, Z. D. The impact of optically rectified fields on plasmonic electrocatalysis. Faraday Discuss. (2019). doi:10.1039/C8FD00135A

21. Cronin, S. B. et al. Hot Electron-driven Photocatalysis and Transient Absorption Spectroscopy in Plasmon Resonant Grating Structures. Faraday Discuss. (2018). doi:10.1039/C8FD00141C

22. Dutta, A. et al. Gap-plasmon enhanced water splitting with ultrathin hematite films: the role of plasmonic-based light trapping and hot electrons. Faraday Discuss. (2019). doi:10.1039/C8FD00148K

23. Miao, P., Ma, Y., Sun, M., Li, J. \& Xu, P. Tuning the SERS activity and plasmon-driven reduction of p-nitrothiophenol on a Ag@MoS 2 film. Faraday Discuss. (2019). doi:10.1039/C8FD00139A

24. Atta, S., Celik, F. E. \& Fabris, L. Enhancing hot electron generation and injection in the near infrared via rational design and controlled synthesis of TiO 2 -gold nanostructures. Faraday Discuss. (2019). doi:10.1039/C8FD00152A

25. Salmón-Gamboa, J. U. et al. Optimizing hot carrier effects in Pt-decorated plasmonic heterostructures. Faraday Discuss. (2019). doi:10.1039/C8FD00150B

26. Thangamuthu, M., Santschi, C. \& Martin, O. J. F. Photocatalytic ammonia production enhanced by a plasmonic near-field and hot electrons originating from aluminium nanostructures. Faraday Discuss. (2019). doi:10.1039/C8FD00146D

27. Bardey, S. et al. Plasmonic photocatalysis applied to solar fuels. Faraday Discuss. (2019). doi:10.1039/C8FD00144H

28. Huang, J. et al. Plasmon-Induced Optical Control over Dithionite-Mediated Chemical Redox 
Reactions. Faraday Discuss. (2018). doi:10.1039/C8FD00155C

29. Anggara, K., Leung, L., Timm, M. J., Hu, Z. \& Polanyi, J. C. Electron-induced molecular dissociation at a surface leads to reactive collisions at selected impact parameters. Faraday Discuss. (2019). doi:10.1039/C8FD00137E

30. Liu, T., Besteiro, L. V., Wang, Z. \& Govorov, A. O. Generation of hot electrons in nanostructures incorporating conventional and unconventional plasmonic materials. Faraday Discuss. (2019). doi:10.1039/C8FD00145F

31. Rahaman, M. et al. The role of a plasmonic substrate on the enhancement and spatial resolution of tip-enhanced Raman scattering. Faraday Discuss. (2019). doi:10.1039/C8FD00142A

32. Lee, H. et al. Enhanced hot electron generation by inverse metal-oxide interfaces on catalytic nanodiode. Faraday Discuss. (2019). doi:10.1039/C8FD00136G

33. Ciccarino, C. J., Chakraborty, C., Englund, D. R. \& Narang, P. Carrier dynamics and spin-valleylayer effects in bilayer transition metal dichalcogenides. Faraday Discuss. (2019). doi:10.1039/C8FD00159F

34. Aguirregabiria, G. et al. Dynamics of electron-emission currents in plasmonic gaps induced by strong fields. Faraday Discuss. (2019). doi:10.1039/C8FD00158H

35. Marini, A., Ciattoni, A. \& Conti, C. Out-of-equilibrium electron dynamics of silver driven by ultrafast electromagnetic fields - a novel hydrodynamical approach. Faraday Discuss. (2019). doi:10.1039/C8FD00153G

36. Cristofolini, P. et al. Coupling Quantum Tunneling with Cavity Photons. Science. 336, 704-707 (2012).

37. Therrien, A. J. et al. Impact of chemical interface damping on surface plasmon dephasing. Faraday Discuss. (2019). doi:10.1039/C8FD00151K 